\title{
Investigation of processed meat consumption from the National Nutritional Survey menuCH and ecological data for colorectal cancer incidence in Switzerland
} \author{
$\underline{\text { Janice Sych }}^{1}$, Ivo Kaelin ${ }^{2}$, Dilara Inanir ${ }_{\text {Sabine Rohrmann }}^{1}{ }^{4}$ Giulia Pestoni ${ }^{4}$, Nadina Mueller ${ }^{1}$ and \\ ${ }^{1}$ Institute of Food and Beverage Innovation, ZHAW School of Life Sciences and Facility Management, Waedenswil, \\ Switzerland, \\ ${ }^{2}$ Institute of Applied Simulation, ZHAW School of Life Sciences and Facility Management, Waedenswil, Switzerland, \\ ${ }^{3}$ Health Department, Bern University of Applied Sciences, Bern, Switzerland and \\ ${ }^{4}$ Division of Chronic Disease Epidemiology, Epidemiology, Biostatistics and Prevention Institute, University of Zurich, \\ Zurich, Switzerland
}

\begin{abstract}
Processed meat (PM) has an important role in diet of the Swiss population, but increasingly regarded as a food group of concern due to epidemiological evidence for its association with colorectal cancer (CRC) and other chronic diseases. Data on CRC incidence for men and women and by region is available in Switzerland, but cannot be linked with PM intake.

This secondary analysis aimed to describe PM consumption in Switzerland, using data from the National Nutritional Survey menuCH, to investigate associations between PM intake and sociodemographic and lifestyle factors, and to examine CRC incidence in Switzerland for any similar regional patterns to PM consumption.

Intakes of total PM and of categories ham, bacon, sausage and other PM types were described by means and standard errors (SEM). Multiple regression analysis was used to investigate associations between meat intake (total meat and PM, assessed separately, $\mathrm{g} / 1000 \mathrm{kcal}$ ) and the following sociodemographic and lifestyle factors: sex, language-speaking region, age-category, nationality, education, gross household income, household status, smoking status, overall health status (self-reported), and currently following a weight-loss diet. Data were weighted for age, sex, marital status, major areas of Switzerland, nationality and household size, and for consumption data, also season and weekday.

Results show PM was consumed by approximately $70 \%$ of the population with mean total intake of 42.7 (SEM $1.2 \mathrm{~g} / \mathrm{d}$ ). Sausage intake was highest with $16.8 \mathrm{~g} / \mathrm{day}$, followed by ham, other PM and bacon, 12.4, 10.6, and $2.0 \mathrm{~g} / \mathrm{day}$, respectively. Sex was significantly associated with total meat and PM intake; women consumed $10.1 \mathrm{~g} / 1000 \mathrm{kcal}$ less total meat [95\% CI: -13.60; -6.64], and $4.70 \mathrm{~g} / 1000$ kcal less PM [95\% CI: $-6.73 ;-2.68]$ than men. For both variables, total meat and PM intake, positive associations were observed for overweight, obesity and current smoking; and negative associations observed for tertiary education and following a diet. The later was significant only for PM intake. The ecological data for CRC incidence revealed much higher rates for men than women, over 24 years; but the data shown by language region did not reveal any particular pattern.

This study is the first to describe intakes of total and different PM types, based on national representative data. Positive associations between PM intake and smoking and obesity merit careful monitoring. More regular data collection by methods enabling separate quantification of meat and PM, as well as relevant health biomarkers are needed in future studies.
\end{abstract}

\section{Conflict of Interest}

There is no conflict of interest. 